\title{
Propagation of Current Waves along Randomly Located Multiconductor Transmission Lines inside a Rectangular Resonator
}

\author{
Sergey V. Tkachenko $\left(\mathbb{D}\right.$, Juergen B. Nitsch, Moustafa Raya $\mathbb{D}^{D}$, and Ralf Vick \\ Otto-von-Guericke-University Magdeburg, IMT, Universitaetsplatz 2, D-39106 Magdeburg, Germany \\ Correspondence should be addressed to Sergey V. Tkachenko; sergey.tkachenko@ovgu.de
}

Received 14 March 2018; Accepted 4 June 2018; Published 4 July 2018

Academic Editor: Nunzio Salerno

Copyright (C) 2018 Sergey V. Tkachenko et al. This is an open access article distributed under the Creative Commons Attribution License, which permits unrestricted use, distribution, and reproduction in any medium, provided the original work is properly cited.

\begin{abstract}
Propagation of current waves along randomly located multiconductor transmission lines inside a rectangular resonator is investigated. Two different line configurations are considered: parallel wires and perpendicular lines. The analytical solutions for both types of lines are obtained, using the method of symmetrical lines inside the resonator. Computer realization of this method allows it to obtain a fast solution. Therefore, it becomes possible to investigate statistical properties of induced currents. It could be shown that the mutual coupling of the currents in the wires is strong when the frequency is near resonances in the system "cavity and wires" and relatively small far from these resonances. Stochastization leads to a broadening of the resonance curves.
\end{abstract}

\section{Introduction}

Transmission lines of different kinds are widely used in different branches of modern technique and industry, especially in power engineering and electronics. They can serve as a channel for the propagation of useful signals and energy, as well as a receiver of different kind of intentional and unintentional electromagnetic interferences. Thus, the study of signal propagation along transmission lines and the external electromagnetic field coupling with transmission lines and wiring systems is an important task of electromagnetic compatibility. This problem is complex itself, especially for the high frequency case, when the transversal dimension of the transmission line is comparable with the wavelength, and also for the treatment of multiconductor lines. Moreover, this task is additionally complicated by the fact that transmission lines are often placed inside resonator-like objects: car bodies, aircraft fuselages, computer cases, shielded rooms, etc. Due to the resonator behavior there is a strong coupling of the penetrated field with the wires inside and vice versa; this can greatly affect the signal propagation on conductors. In addition, often the exact positions of the lines and the corresponding electrical parameters per-unit length are unknown and have randomly distributed values. This leads to the necessity to study stochastic transmission lines in the resonator, including the more complex multiconductor variant.

In literature, there are a number of papers which deal with stochastic cavities, i.e., with cavities whose parameters are only known stochastically. This activity is mainly connected with the practical necessity to study Mode Stirred Chambers (MSC) and to clarify the correspondence between measurements in a MSC and in free space. For those studies papers of S. Hemmadi and T.M. Antonsen and their groups [1, 2] are well known, which are based on a deep investigation of the mathematical properties of stochastic cavities [3], when they investigate the response of components in stochastic cavities. The authors of [4] consider stochastically located small antennas to create stochastic fields in a MSC. Other papers (see, e.g., [5-7]) are devoted to the investigation of coupling of a random electromagnetic field to deterministic transmission lines inside stochastic cavities (MSC).

In contrast, we are interested in stochastic lines inside the resonator with deterministic parameters. This view is linked to the need for a statistical analysis of the vulnerability of electronic devices to intentional and nonintentional 


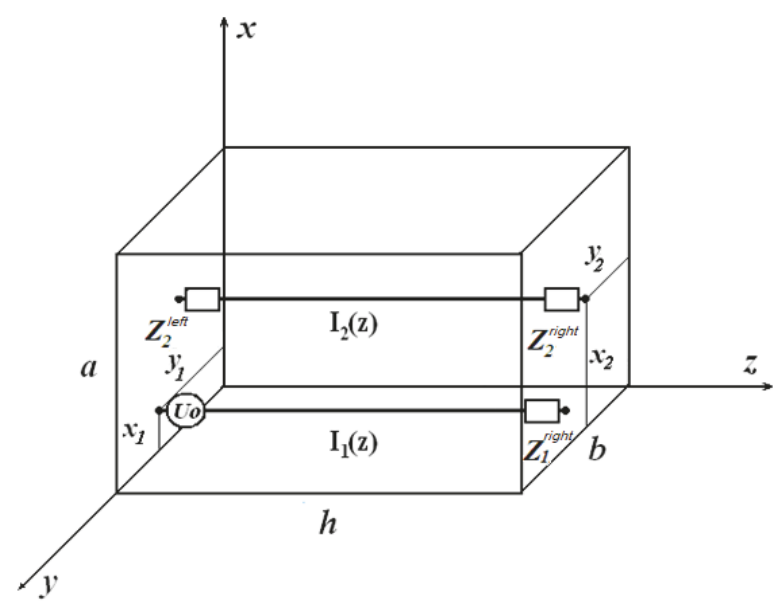

FIGURE 1: Two-wire symmetrically loaded line in a resonator.

interferences, mutual coupling interferences, etc. We only know one paper which deals with a single-wire line of stochastic geometry inside a resonator [8]. In [8], the transmission line is considered inside a metallic rectangular box with a rectangular aperture. The system is excited by an incident plane wave on the aperture. The transmission line has a fixed length and positions of connectors but different layouts (or pats) for each statistical realization. The finitedifference time-domain method (FDTD) and fast Fourier transform were used to obtain the voltages at the terminals and their statistical parameters: average value and dispersion. It was shown that maxima of these averaged voltages occurred next to the cavity resonances. It is physically obvious that the dispersion of these values is defined by the stochastic characteristic of the line geometry. However, the direct numerical methods are very time consuming. Therefore in [8] only 12 statistical realizations were chosen. It seems to be clear that for a deep investigation of this model some analytical or analytic-numerical method has to be developed.

Recently, we proposed an exact solution method for a loaded line in a resonator, which can be excited by an arbitrary electromagnetic field $[9,10]$. From the computational point of view, the solution is reduced to the calculation of a double modal sum with 200-300 terms for each sum direction and for each considered frequency. This method was applied to a multiloaded line or to two parallel lines [11] excited by a lumped source(s) and by distributed sources, such as additional internal radiating antennas or by EM fields which penetrated into the resonator through apertures [12].

Note that our method is applicable to a line with geometric symmetry of the resonator: the line connects two opposite rectangular walls and it is parallel to the four other walls (see Figure 1). Nevertheless, we have applied this method for the investigation of stochastic lines inside the resonator.

In $[13,14]$, one single-wire line in the resonator was considered. The stochastization was created by multiple loading with random positions along the line or by random values of the reactive loads. With an appropriate choice of parameters, this model can serve to describe a line with stochastic geometry. It was shown that for a stochastic line in the resonator, unlike in free space, the current wave on the "average" passes through the transmission line. This is explained by the multiple rereflection of the signal from walls of the resonator. Also, it was shown that the PDF of the absolute value of the transmission coefficient for current wave for the case of strong stochastization (when the average value of transmission coefficient equals zero) can be described by the binominal distribution, as the consequence of the principle of maximum entropy.

In the present paper we extend the method $[9,10]$ to multiconductor lines in the resonator. Stochastization is created by random positions of the lines. The rest of the paper is organized as follows: In Section 2 we derive equations for the EM coupling of parallel multiconductor loaded lines in a rectangular resonator. Then the corresponding solution will be applied to the lines with random transverse coordinates and for the calculation of the corresponding lower statistical moments. In Section 3 we consider two perpendicular wires in the resonator. Again, the general equations for the currents induced in both wires for arbitrary excitations are obtained. Unlike the previous case, the symmetry of the problem is not conserved. The modal matrix equations which describe the induced currents do not split. For a solution operations with infinite matrices are required. However, for a relatively small number of accounted modes (about 300-500) one can obtain the solution quite fast. The obtained solutions again are applied for the investigation of statistical moments of the induced currents for random positions of the lines. Section 4 concludes the paper.

\section{Multiconductor Transmission Lines inside a Rectangular Resonator}

2.1. Exact Analytical Solution of EFIE for Short-Circuited Lines. We consider $\mathrm{N}$ parallel wires inside a rectangular resonator with sides a, b, and h. The wires keep the symmetry of the resonator: they connect two opposite walls of the resonator and they are parallel to all other walls and to the $\mathrm{z}$ axis (see Figure 1). The positions of the $\mathrm{n}^{\text {th }}$ wire in the xy plane is given by $\vec{\rho}_{n}=\left(x_{n}, y_{n}\right)$. It is assumed that there is an electromagnetic field $\vec{E}^{\text {ex }}(\vec{r})$ inside the resonator which is excited by one or another way: by an internal antenna, by penetration through an aperture, or by a lumped source. This field induces currents $I_{1}(z), I_{2}(z), \ldots I_{N}(z)$ in the conductors. The currents are represented as a column vector

$$
\mathbf{I}(\mathbf{z}):=\left[I_{1}(z), I_{2}(z), \ldots I_{N}(z)\right]^{T}
$$

which are, in turn, the source of a scattered field, $\vec{E}^{s c}(\vec{r})$. Further, assuming that all wires are thin-the radius of the each wire $r_{0}$ is essentially smaller than all linear dimensions of the problem including wavelength $\lambda$-we can apply so called thin-wire approximation. In this approximation only axial components of the current are taken into account. Moreover, it is assumed that all current densities are concentrated along the wire axis; however, the zero-boundary conditions for the 
perfectly conducting wires have to be satisfied for the total tangential electric field on the boundary of the each wire $S_{n}$.

$$
\left(\vec{E}_{z}^{i n}(\vec{r})+\vec{E}_{z}^{s c}(\vec{r})\right)_{\vec{r} \in S_{n}}=0, \quad n=1,2, \ldots N
$$

The scattered field in the considered geometry can be calculated using $\mathrm{zZ}$ components of the resonator's Green's function "electrical current->electrical field", $\overline{\bar{G}}_{z z}^{E}$. Thus, we can establish a system of integrodifferential equations that determine the induced current in the wires:

$$
\begin{aligned}
& \sum_{n_{1}=1}^{N} \int_{0}^{h} \overline{\bar{G}}_{z z}^{E}\left(x_{n}+r_{0}, y_{n}, z, x_{n_{1}}, y_{n_{1}}, z_{1}, \omega\right) I\left(z_{1}\right) d z_{1} \\
& \quad+E_{z}^{e x}\left(x_{n}+r_{0}, y_{n}, z\right), \quad n=1,2, \ldots N
\end{aligned}
$$

The zz component Green function for the electric field, in turn, can be evaluated from the $\mathrm{zz}$ component of Green's function for the vector potential $\overline{\bar{G}}_{z z}^{A}$ :

$$
\begin{aligned}
\overline{\bar{G}}_{z z}^{E}\left(\vec{r}_{1}, \vec{r}_{2}, \omega\right) & =\frac{1}{j \omega \varepsilon_{0} \mu_{0}}\left(k^{2}+\frac{\partial^{2}}{\partial z^{2}}\right) \overline{\bar{G}}_{z z}^{A}\left(\vec{r}_{1}, \vec{r}_{2}, \omega\right) \\
\text { where } G_{z z}^{A}\left(\vec{r}, \vec{r}_{1}\right) & =\frac{4 \mu_{0}}{V} \sum_{\substack{m_{x}=1 \\
m_{y}=1 \\
m_{z}=0}}^{\infty} \frac{\varepsilon_{m_{3}} \sin \left(k_{x}^{v} x_{1}\right) \sin \left(k_{x}^{v} x\right) \sin \left(k_{y}^{v} y_{1}\right) \sin \left(k_{y}^{v} y\right) \cos \left(k_{z}^{v} z_{1}\right) \cos \left(k_{z}^{v} z\right)}{\left(k^{v}\right)^{2}-k^{2}+j \delta \operatorname{sign}(k)}
\end{aligned}
$$

We have introduced the following notations:

$$
\begin{aligned}
k_{x}^{v} & =\pi \frac{m_{x}}{a}, \\
k_{y}^{v} & =\pi \frac{m_{y}}{b}, \\
k_{z}^{v} & =\pi \frac{m_{z}}{h}, \\
k^{v} & =\sqrt{\left(k_{x}^{v}\right)^{2}+\left(k_{y}^{v}\right)^{2}+\left(k_{z}^{v}\right)^{2},} \\
\text { and } \varepsilon_{m} & = \begin{cases}1, & \text { if } m=0 \\
2 & \text { if } m \neq 0 .\end{cases}
\end{aligned}
$$

In the denominator of (5) the summand $j \delta \operatorname{sign}(k)$ with small $\delta>0$ shifts the pole $k^{2}=k_{v}^{2}$ in the complex frequency plane in the upper half plane of the complex variable $k:=\omega / c$. This is necessary, e.g., during the calculation of the system response in time domain by the inverse Fourier transform, when one has to perform the integration in the complex plane by applying the residue theorem. In the paper [15], e.g., this small quantity $\delta$ is assigned a physical interpretation, e.g., small radiation losses through an aperture or small losses in the walls. In other words, this quantity is connected with quality factor of the resonator Q by $\delta=k^{2} / Q$ [16]. Since further in this paper we will only consider resonators without losses in the frequency domain, the factor $\delta$ will be omitted.

Thus, one can write the function $\overline{\bar{G}}_{z z}^{E}$ as

$$
\begin{aligned}
& G_{z z}^{E}\left(\vec{r}, \vec{r}_{1}\right)=\frac{\eta_{0}}{j k h} \sum_{\substack{m_{x}=1 \\
m y=1 \\
m z=0}}^{\infty} \varepsilon_{m_{3}}\left(k^{2}-\left(k_{z}^{v}\right)^{2}\right) \\
& \cdot S\left(k, m_{z}, x, y, x_{1}, y_{1}\right) \cos \left(k_{z}^{v} z_{1}\right) \cos \left(k_{z}^{v} z\right)
\end{aligned}
$$

where we introduced the notation for the double sum:

$$
\begin{aligned}
& S\left(k, m_{z}, x, y, x_{1}, y_{1}\right) \\
& :=\frac{4}{a b} \sum_{\substack{m_{x}=1 \\
m_{y}=1}}^{\infty} \frac{\sin \left(k_{x}^{v} x\right) \sin \left(k_{x}^{v} x_{1}\right) \sin \left(k_{y}^{v} y\right) \sin \left(k_{y}^{v} y_{1}\right)}{k_{v}^{2}-k^{2}}
\end{aligned}
$$

First we consider short-circuited wires (see Figure 1, where all loads are zero), where the induced currents satisfy the zero Neumann boundary conditions: $\partial I_{n}(z) /\left.\partial z\right|_{z=0}=$ $\partial I(z) /\left.\partial z\right|_{z=h}=0$ with $\mathrm{n}=1,2 . . \mathrm{N}$. This allows decomposing each current into the Fourier transform:

$$
\begin{array}{r}
I_{n}(z)=\sum_{m_{z}=0}^{\infty} I_{n}\left(m_{z}\right) \cdot \cos \left(\frac{m_{z} \pi z}{h}\right) \\
\text { or } \mathbf{I}(z)=\sum_{m_{z}=0}^{\infty} \mathbf{I}\left(m_{z}\right) \cdot \cos \left(\frac{m_{z} \pi z}{h}\right)
\end{array}
$$

Then we decompose the exciting electrical field into a Fourier series for each wire in (3). (One can make this decomposition due to the zero boundary conditions for the tangential components of electrical field on the walls)

$$
\begin{aligned}
& E_{z}^{e x}\left(x_{n}, y_{n} z\right)=\sum_{m_{3}=0}^{\infty} E_{z}^{e x}\left(x_{n}, y_{n}, m_{z}\right) \cos \left(\frac{m_{z} \pi z}{h}\right) \\
& \text { or } \mathbf{E}_{z}^{e x}(z)=\sum_{m_{3}=0}^{\infty} \mathbf{E}_{z}^{e x}\left(m_{z}\right) \cos \left(\frac{m_{z} \pi z}{h}\right) \\
& \quad \mathbf{E}_{\mathbf{z}}^{\text {ex }}(z) \\
& \quad:=\left(\left[E_{z}^{e x}\left(\vec{\rho}_{1}, z\right), E_{z}^{e x}\left(\vec{\rho}_{2}, z\right), \ldots E_{z}^{e x}\left(\vec{\rho}_{N}, z\right)\right]^{T}\right), \\
& \left.\quad:=\left(\left[E_{z}^{e x}\left(m_{z}\right), z\right), E_{z}^{e x}\left(m_{z}, z\right), \ldots E_{z}^{e x}\left(m_{z}, z\right)\right]^{T}\right)
\end{aligned}
$$


where the Fourier coefficients (10a), (10b) can be obtained by integration:

$$
\begin{aligned}
& E_{z}^{e x}\left(x_{n}, y_{n}, m_{z}\right) \\
& \quad=\frac{h}{\varepsilon_{m_{z}, 0}} \int_{0}^{h} E_{z}^{e x}\left(x_{n}, y_{n}, z\right) \cos \left(\frac{m_{z} \pi z}{h}\right) d z, \\
& \mathbf{E}_{\mathbf{z}}^{\mathbf{e x}}\left(m_{z}\right)=\frac{h}{\varepsilon_{m_{z}, 0}} \int_{0}^{h} \mathbf{E}_{\mathbf{z}}^{\mathbf{e x}}(z) \cos \left(\frac{m_{z} \pi z}{h}\right) d z
\end{aligned}
$$

Then, substituting (9a), (9b), (10a), (10b), and (7)-(8) into (3) and taking into account the orthogonality of the cosine functions, we can obtain the linear system for the Fourier images of the unknown currents:

$$
\begin{aligned}
& E_{z}^{e x}\left(x_{n}, y_{n}, m_{z}\right)+\frac{\eta_{0}}{j k}\left(k^{2}-\left(k_{z}^{v}\right)^{2}\right) \\
& \cdot \sum_{n=1}^{N} S\left(k, m_{z}, x_{n}+r_{0}, y_{n}, x_{n_{1}}, y_{n_{1}}\right) I_{n_{1}}\left(m_{z}\right)=0
\end{aligned}
$$

Introducing the matrix of two-dimensional scattering $\mathbf{S}\left(k, m_{z}\right)$, we can rewrite (13) in the matrix form:

$$
\mathbf{E}_{\mathbf{z}}^{\mathbf{e x}}\left(m_{z}\right)+\frac{\eta_{0}}{j k}\left(k^{2}-\left(k_{z}^{v}\right)^{2}\right) \mathbf{S}\left(k, m_{z}\right) \cdot \mathbf{I}\left(m_{z}\right)=0
$$

where $\left[\mathbf{S}\left(k, m_{z}\right)\right]_{n, n_{1}}=S\left(k, m_{z}, x_{n}+r_{0}, y_{n}, x_{n_{1}}, y_{n_{1}}\right)$.

Then, in the definition of the function $S\left(k, m_{z}, \vec{\rho}, \vec{\rho}_{1}\right)$ (8) one summation can be done analytically (see [17]) and, after some manipulations, we can write expressions for the diagonal and nondiagonal elements of the matrix $\mathbf{S}\left(k, m_{z}\right)$, as a one-dimensional sum [9]:

$$
\begin{aligned}
& {\left[\mathbf{S}\left(k, m_{z}\right)\right]_{n, n_{1}}=\frac{2}{a} \sum_{m_{x}=1}^{\infty} \sin \left(k_{x}^{v} x_{n}\right) \sin \left(k_{x}^{v} x_{1}\right)} \\
& \cdot \frac{\sinh \left(\widetilde{\gamma}_{v}\left(b-y_{>}\right)\right) \sinh \left(\widetilde{\gamma}_{v} y_{<}\right)}{\widetilde{\gamma}_{v} \sinh \left(\widetilde{\gamma}_{v} b\right)}, \text { for } n \neq n_{1} \\
& {\left[\mathbf{S}\left(k, m_{z}\right)\right]_{n, n}=\frac{1}{a} \sum_{m_{x}=1}^{\infty} \sin ^{2}\left(k_{x}^{v} x_{n}\right)} \\
& \cdot\left[\frac{2 \sinh \left(\widetilde{\gamma}_{v}\left(b-y_{n}\right)\right) \sinh \left(\widetilde{\gamma}_{v} y_{n}\right)}{\widetilde{\gamma}_{v} \sinh \left(\widetilde{\gamma}_{v} b\right)}-\frac{1}{k_{v x}}\right]+\frac{1}{2 \pi} \\
& \cdot \ln \left[\frac{2 a\left|\sin \left(\pi x_{n} / a\right)\right|}{\pi r_{0}}\right] \\
& y_{>}:=\max \left(y_{n}, y_{n_{1}}\right), \\
& y_{<}:=\min \left(y_{n}, y_{n_{1}}\right), \\
& \widetilde{\gamma}_{v}:=\sqrt{\left(k_{x}^{v}\right)^{2}+\left(k_{z}^{v}\right)^{2}-k^{2} .}
\end{aligned}
$$

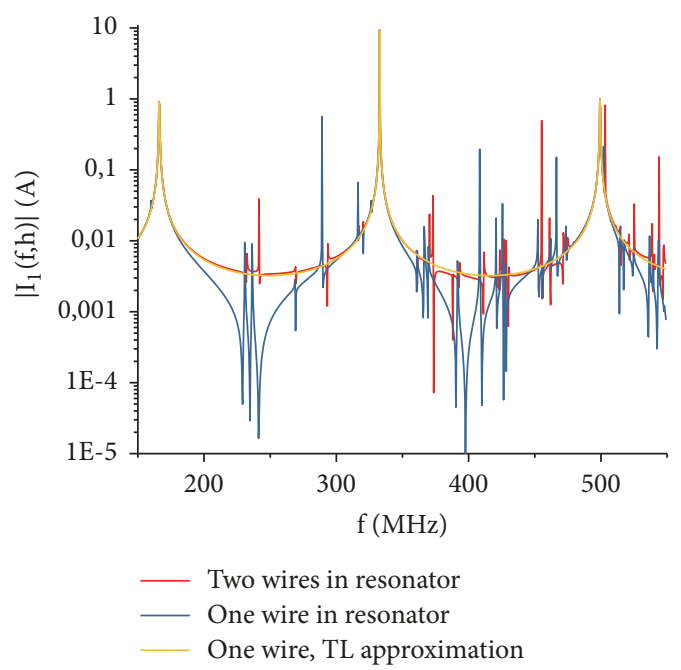

FIGURE 2: Deterministic parallel lines in a resonator. Response at the end of the first line calculated by TL approximation and the exact solution with and without accounting for the second line.

The solutions for the modal amplitude and the $\mathrm{z}$-dependence of the vector-current can be obtained using (13), (14), and (15):

$$
\begin{aligned}
& \mathbf{I}\left(m_{z}\right)=\frac{j k}{\eta_{0}} \frac{1}{\left(k_{z}^{v}\right)^{2}-k^{2}} \mathbf{S}^{-1}\left(k, m_{z}\right) \cdot \mathbf{E}_{\mathbf{z}}^{\mathbf{e x}}\left(m_{z}\right), \\
& \mathbf{I}(z)=\frac{j k}{\eta_{0}} \sum_{m_{z}=0}^{\infty} \frac{\cos \left(m_{z} \pi z / h\right)}{\left(k_{z}^{v}\right)^{2}-k^{2}} \mathbf{S}^{-1}\left(k, m_{z}\right) \cdot \mathbf{E}_{\mathbf{z}}^{\mathbf{e x}}\left(m_{z}\right)
\end{aligned}
$$

With the aid of (3), (17a) and (17b) can be rewritten as follows:

$$
\mathbf{I}(z)=\int_{0}^{h} \mathbf{Y}\left(k, z, z_{1}\right) \cdot \mathbf{E}_{\mathbf{z}}^{\mathbf{e x}}\left(z_{1}\right) d z_{1}
$$

where we introduced Green's function matrix for the multiconductor transmission line

$$
\begin{aligned}
& \mathbf{Y}\left(k, z, z_{1}\right):=\frac{j k}{\eta_{0} h} \\
& \quad \sum_{m_{z}=0}^{\infty} \frac{\varepsilon_{m_{z}, 0} \cos \left(m_{z} \pi z / h\right) \cos \left(m_{z} \pi z_{1} / h\right)}{\left(k_{z}^{v}\right)^{2}-k^{2}} \mathbf{S}^{-1}(k, \\
& \left.\quad m_{z}\right)
\end{aligned}
$$

Equations (18)-(19) together with (15)-(16) yield the solution for the current in the short- circuited multiconductor transmission line for an arbitrary exciting field. One can show that if the frequency is far from cavity resonances and the wires are close to one of the cavity walls, these equations yield the classical TL approximation solution for the short-circuited multiconductor line obtained by Fourier transformation.

In Figures 2-4 some numerical examples for deterministic parallel lines in a resonator are shown. The dimensions of the resonator are $\mathrm{a}=1.5 \mathrm{~m}, \mathrm{~b}=1.2 \mathrm{~m}$, and $\mathrm{h}=0.9 \mathrm{~m}$. Transverse coordinates of the first line are $x_{1}=9 \mathrm{~cm}, y_{1}=37 \mathrm{~cm}$. The first line is fed by a lumped source with unit amplitude $\mathrm{U}_{0}=1 \mathrm{~V}$, 


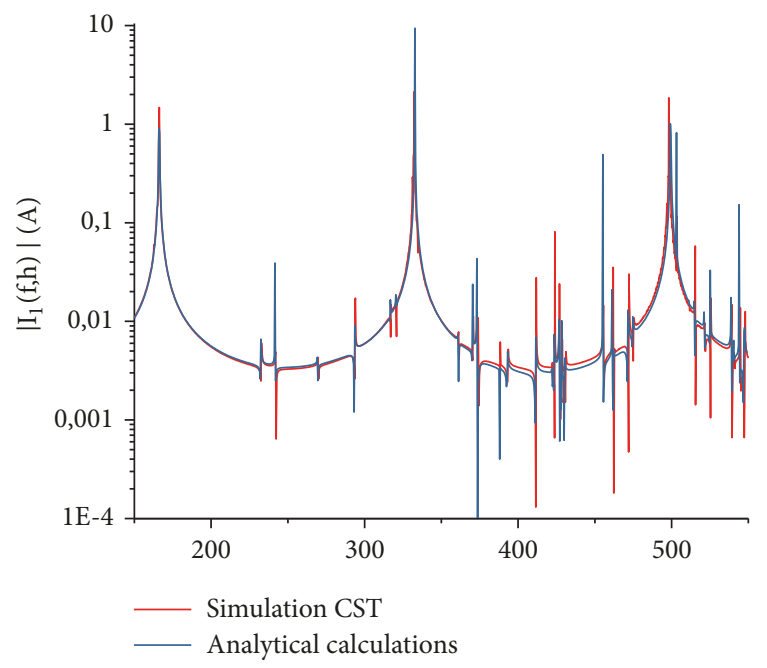

FIGURE 3: Deterministic parallel lines in resonator. Response at the end of the first line calculated by the exact analytical solution with CST code.

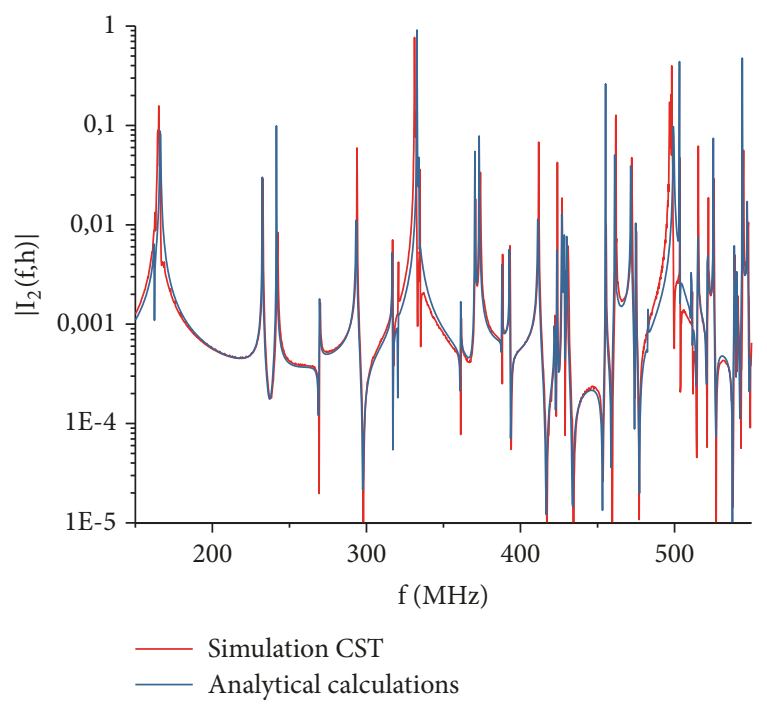

FIGURE 4: Deterministic parallel lines in resonator. Response at the end of the second line calculated by the exact analytical solution with and CST code.

located at the left terminal and short-circuited both at both ends. (For the numerical simulation we used the values $Z^{\text {left }}{ }_{1}=$ $\mathrm{Z}^{\text {right }}{ }_{1}=0.2 \Omega$ ) The transverse coordinates of the second wire are $x_{1}=20 \mathrm{~cm}, y_{1}=50 \mathrm{~cm}$. The second wire is also shortcircuited at left and right terminal.

(For the numerical simulation we used the values $\mathrm{Z}^{\text {left }}{ }_{2}=$ $\mathrm{Z}^{\text {right }}{ }_{2}=0.2 \Omega$.) The radius of both wires is $\mathrm{r}_{0}=1 \mathrm{~mm}$. In Figure 2 the current at the right terminal of the first line is presented, calculated by an analytical method with and without accounting for the second wire, also in the transmission line approximation [9]. In Figures 3 and 4 the currents at the right terminals of the first and second wire are shown, respectively, together with a comparison of the results obtained by the code
CST MICROWAVE STUDIO [18]. Since the model includes a resonator, the frequency domain solver has been used based on the finite element method (FEM). From the analysis of the calculations one can observe the following: The analytical method for the resonator and the direct numerical calculation yield a good agreement for the considered system. Far from the cavity resonances the current in the second, passive wire is smaller in comparison to the current in the first one, but near the resonances they can have the same order of magnitude. In turn, the influence of the second wire on the first one is quite small for a frequency interval far from cavity resonances. In this case the current in the first wire can be described by the usual TL approximation. At the same time, near the resonances the influence of the second wire is essential. The response of the first wire alone and in the presence of the second one is strongly different.

2.2. Exact Analytical Solution for MTL: Accounting for Lumped Excitations and Loads. Consider now the excitation of the transmission line by lumped sources which have the same coordinate $\mathrm{z}_{1}$, but different amplitudes:

$$
\begin{aligned}
\mathbf{E}_{z}^{e x}(z) & =\mathbf{U}_{0} \delta\left(z-z_{1}\right), \\
\text { where } \mathbf{U}_{0} & =\left[U_{01}, U_{02}, \ldots U_{0 N}\right]^{T}
\end{aligned}
$$

Then, from (18) the current is given by

$$
\mathbf{I}(z)=\mathbf{Y}\left(k, z, z_{1}\right) \cdot \mathbf{U}_{0}
$$

Now it is possible to take into account also lumped impedance at the left and right terminals of the line. The lumped loads at the left terminal at the point $\mathrm{z}_{1}=\Delta(\Delta \longrightarrow 0)$ can be considered as controlled lumped voltage sources with unknown amplitudes:

$$
\begin{aligned}
E_{z}^{Z_{\text {left }}}\left(x_{n}, y_{n}, z\right) & =-Z_{n}^{\text {left }} \cdot I_{n}\left(z_{1}\right) \delta\left(z-z_{1}\right) \\
\text { or } \mathbf{E}_{z}^{Z_{\text {left }}}(z) & =-\mathbf{Z}^{\text {left }} \cdot \mathbf{I}\left(z_{1}\right) \delta\left(z-z_{1}\right)
\end{aligned}
$$

where $\mathbf{Z}^{\text {left }}=\operatorname{diag}\left[Z_{n}^{\text {left }}\right]$.

The same trick can be done for the lumped loads at the right terminal $\mathrm{z}_{2}=\mathrm{h}-\Delta(\Delta \longrightarrow 0)$ :

$$
\begin{gathered}
E_{z}^{Z_{\text {right }}}\left(x_{n}, y_{n}, z\right)=-Z_{n}^{\text {right }} \cdot I_{n}\left(z_{2}\right) \delta\left(z-z_{2}\right) \\
\quad \text { or } \mathbf{E}_{z}^{Z_{\text {rifht }}}(z)=-\mathbf{Z}^{\text {right }} \cdot \mathbf{I}\left(z_{2}\right) \delta\left(z-z_{2}\right)
\end{gathered}
$$

where $\mathbf{Z}^{\text {right }}=\operatorname{diag}\left[Z_{n}^{\text {right }}\right]$.

Then, the column vector for total exciting field for the loaded multiconductor line with lumped sources at the left terminal is

$$
\begin{aligned}
\mathbf{E}_{z}^{\text {tot }}(z)= & \mathbf{U}_{0} \delta\left(z-z_{1}\right)-\mathbf{Z}^{\text {left }} \cdot \mathbf{I}\left(z_{1}\right) \delta\left(z-z_{1}\right) \\
& -\mathbf{Z}^{\text {right }} \cdot \mathbf{I}\left(z_{2}\right) \delta\left(z-z_{2}\right)
\end{aligned}
$$

which yields matrix equation for the current:

$$
\begin{aligned}
\mathbf{I}(z)= & \mathbf{Y}\left(k, z, z_{1}\right) \cdot\left(\mathbf{U}_{0}-\mathbf{Z}^{\text {left }} \cdot \mathbf{I}\left(z_{1}\right)\right)-\mathbf{Y}\left(k, z, z_{2}\right) \\
& \cdot \mathbf{Z}^{\text {right }} \cdot \mathbf{I}\left(z_{2}\right)
\end{aligned}
$$




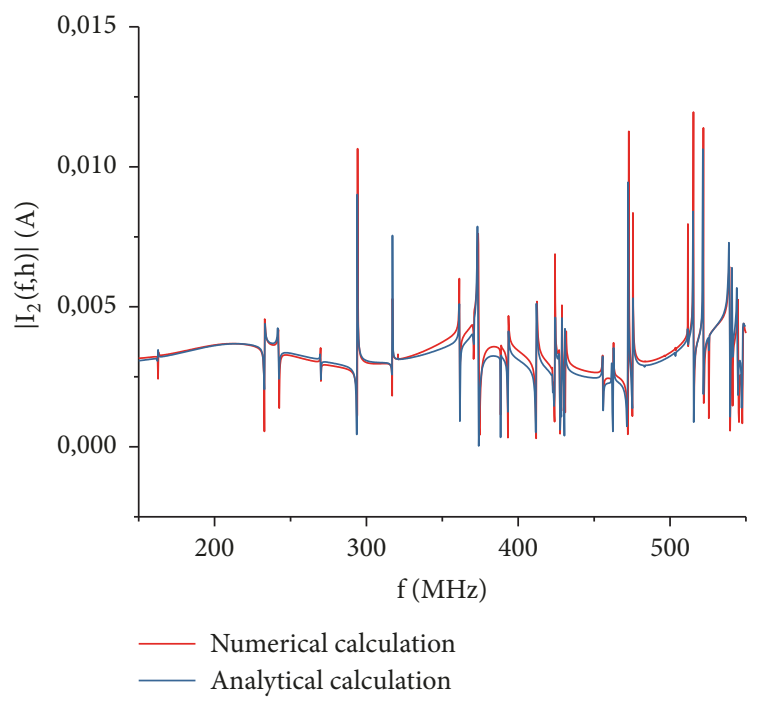

FIGURE 5: Deterministic parallel lines in resonator. Response at the end of the first, loaded, line calculated by the exact analytical solution and by the CST code.

After that calculating column vector of currents at the points $\mathrm{z}_{1}$ and $\mathrm{z}_{2}$, we obtain linear system (26) for the values $\mathbf{I}\left(z_{1}\right)$ and $\mathrm{I}\left(z_{2}\right)$, which, in turn, defines current in arbitrary point by $(25)$ :

$$
\begin{aligned}
& {\left[\begin{array}{cc}
\mathbf{Y}\left(k, z_{1}, z_{1}\right) \cdot\left(\mathbf{E}+\mathbf{Z}^{\text {left }}\right), & \mathbf{Y}\left(k, z_{1}, z_{2}\right) \cdot \mathbf{Z}^{\text {right }} \\
\mathbf{Y}\left(k, z_{2}, z_{1}\right) \cdot \mathbf{Z}^{\text {left }}, & \mathbf{Y}\left(k, z_{2}, z_{2}\right) \cdot\left(\mathbf{E}+\mathbf{Z}^{\text {right }}\right)
\end{array}\right]} \\
& \cdot\left[\begin{array}{l}
\mathbf{I}\left(z_{1}\right) \\
\mathbf{I}\left(z_{2}\right)
\end{array}\right]=\left[\begin{array}{l}
\mathbf{Y}\left(k, z_{1}, z_{1}\right) \cdot \mathbf{U}_{\mathbf{0}} \\
\mathbf{Y}\left(k, z_{2}, z_{1}\right) \cdot \mathbf{U}_{\mathbf{0}}
\end{array}\right]
\end{aligned}
$$

Below we presented a numerical example for the response of the loaded lines (see Figures 5 and 6). We consider a system with the same parameters resonator and lines, except the nonzero load at the right terminal of the first line. It is loaded by the matched load for the single line for TEM waves in resonator: $Z_{1}^{\text {right }}=Z_{C 1}=\left(\eta_{0} / 2 \pi\right) \ln \left[2 x_{1} y_{1} / r_{0} \sqrt{x_{1}^{2}+y_{1}^{2}}\right] \approx$ $309 \Omega$.

As in a previous example, the comparison has shown a good agreement between developed theory and numerical simulation. Far from the resonances current in the first wire is approximately constant, i.e., has TL structure (see $[9,10])$. Also, as in previous example, the mutual influence of the wires is strong near resonances. Note that near the resonances current in the second wire can be comparable and even more than the current in the first wire.

2.3. Randomly Located Multiconductor-Transmission Line. In this section to realize stochastization of multiconductor TL, we consider random positions of the parallel wires. We consider a similar resonator with dimensions $\mathrm{a}=1.5 \mathrm{~m}, \mathrm{~b}=1.2$ $\mathrm{m}$, and $\mathrm{h}=0.9 \mathrm{~m}$. As in the previous case, the first line has a left lumped source; both lines have zero loads at both terminals.

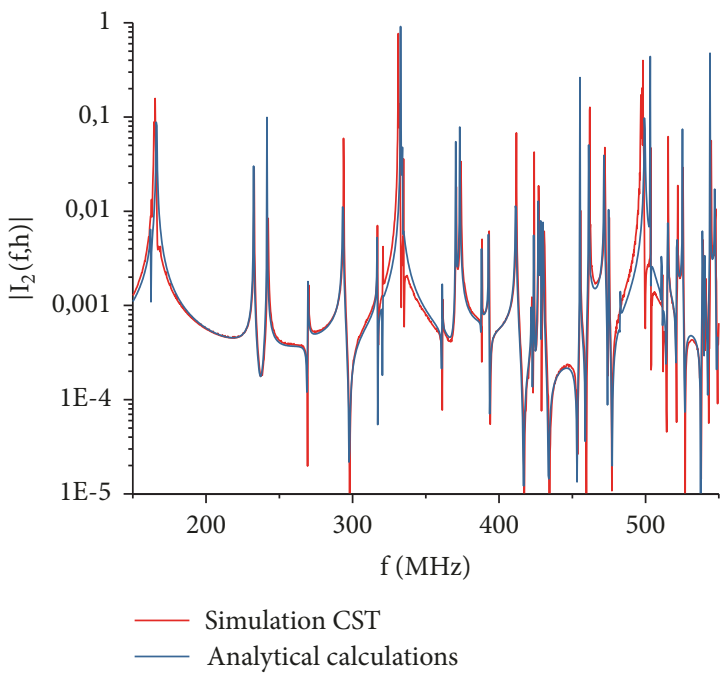

FIGURE 6: Deterministic parallel lines in resonator. Response at the end of the second, short-circuited, line calculated by the exact analytical solution and by the CST code.

The radiuses of the wires are $1 \mathrm{~mm}$. The transversal positions of the both wires in the plane $(\mathrm{x}, \mathrm{y})$ are defined by

$$
\begin{aligned}
& x_{01}=a \cdot(0.05+0.9 \cdot r), \\
& y_{01}=b \cdot(0.05+0.9 \cdot r) \\
& x_{02}=a \cdot(0.05+0.9 \cdot r), \\
& y_{02}=b \cdot(0.05+0.9 \cdot r)
\end{aligned}
$$

where $\mathrm{r}$ is a stochastic value uniformly distributed in the interval $0 \leq r \leq 1$.

The simulation was carried out in the frequency band $150-550 \mathrm{MHz}$ with 500 frequency points. For each frequency point we made 100 statistical events. All calculation required about 4 hours on the computer HP ENVY 17 Notebook PC with processor Intel(R) Core(TM) i7-5500U@ $2.40 \mathrm{GHz}$. Code was realized on the Fortran programming language.

The results of simulations for the right terminal of the first (active) and the second (receiving) wires are represented at Figures 7-10 both for the average absolute value of the induced current for unit source (response function) and for the variance of these values. For comparison, also we present response functions for the deterministic lines with parameters $\mathrm{x}_{1}=9 \mathrm{~cm}, \mathrm{y}_{1}=39 \mathrm{~cm}, \mathrm{x}_{2}=20 \mathrm{~cm}$, and $\mathrm{y}_{2}=50$ $\mathrm{cm}$. From the analysis of the curves one can conclude the following. For the active wire the stochastization changes the positions of the response curves near cavity resonances (system cavity + two wires), but not near the transmission line resonances (see Figure 7). For the receiving wire the influence of stochastization is more strong, but, in principle, all features of previous case are kept (see Figure 9). The statistical variance of both response functions, for the active and receiving wires, has the same order of magnitude: see Figures 8 and 10. Note that from the relative small number 


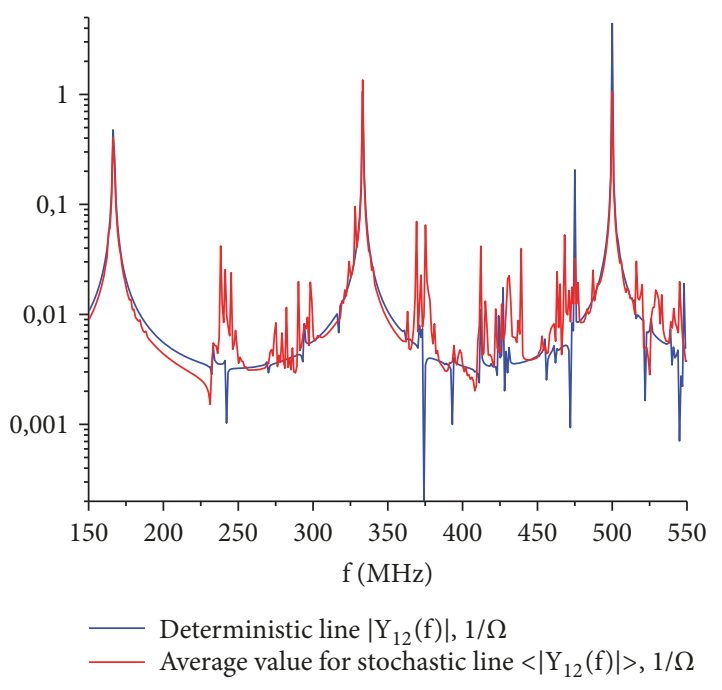

FIGURE 7: Stochastic parallel lines in resonator. Averaged response function at the end of the first, active, line compared with the deterministic result.

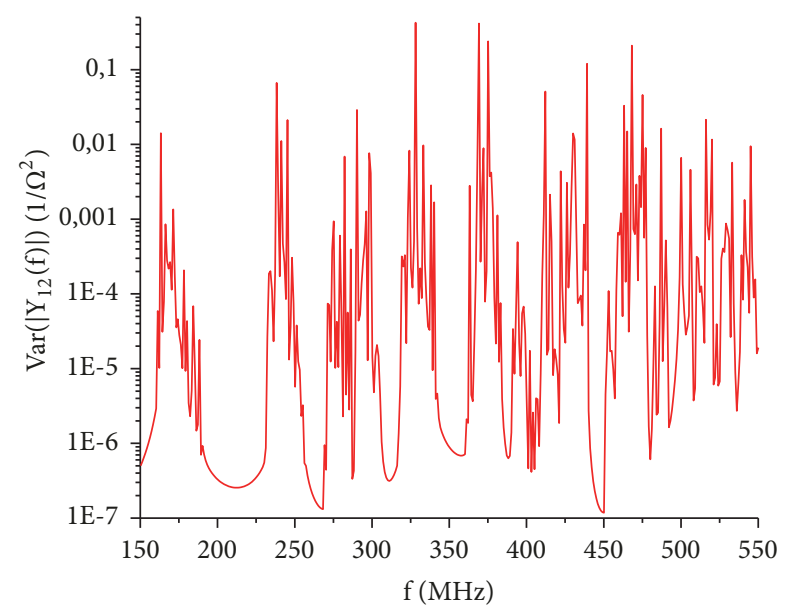

Figure 8: Stochastic parallel lines in resonator. Variance of the response function of the first, active line.

of statistical events the frequency dependence of the average values and variances are not smooth.

\section{Two Perpendicular Wires inside Rectangular Resonator}

3.1. Exact Analytical Solution of EFIE for Short-Circuited Perpendicular Lines. In the present section, we consider another configuration of multiconductor lines with symmetrical geometry in rectangular resonator, which can be interesting for the practical applications on the one hand and allows the analytical consideration on the other hand. Namely, we consider two perpendicular lines in resonator (see Figure 11).

As in the previous case, we expand the exciting tangential electrical fields and induced currents into the Fourier series,

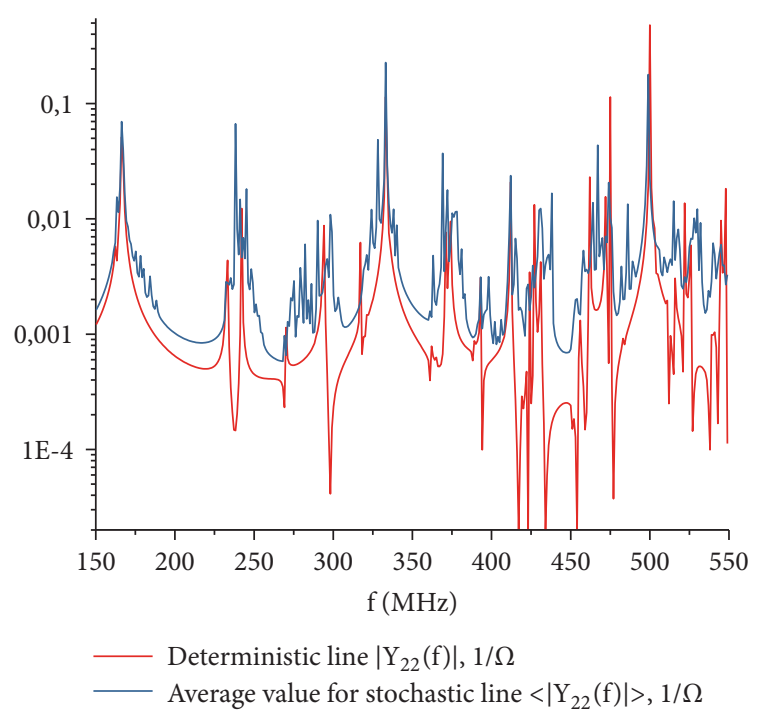

Figure 9: Stochastic parallel lines in resonator. Averaged response function at the end of the second, receiving, line compared with the deterministic result.

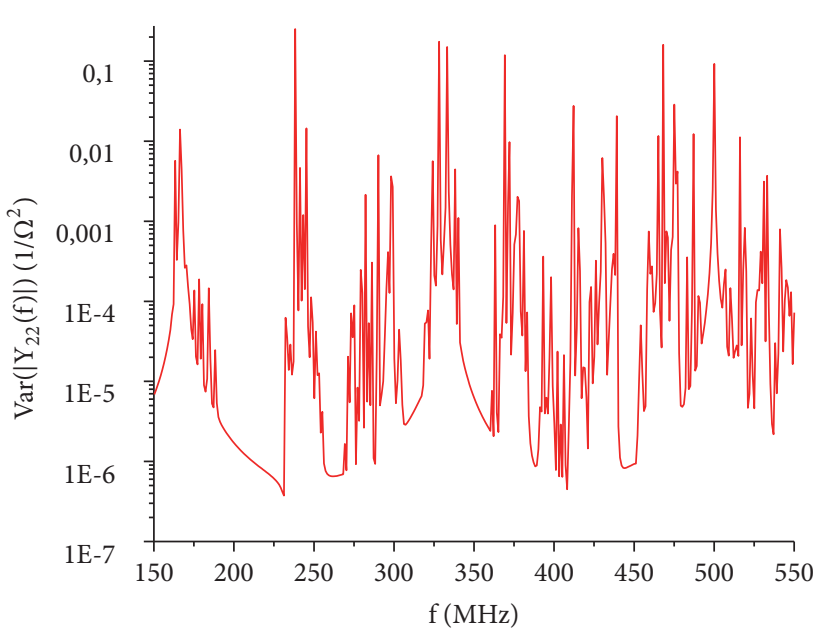

FIGURE 10: Stochastic parallel lines in resonator. Variance of the response function of the second, receiving, line.

but now the basic cosines functions are different for the wires of different directions:

$$
\begin{aligned}
E_{z}^{e x}(x, y, z) & =\sum_{m_{z}=0}^{\infty} E_{z}^{e x}\left(x, y, m_{z}\right) \cos \left(\frac{m_{z} \pi}{h} z\right) \\
E_{x}^{e x}(x, y, z) & =\sum_{m_{x}=0}^{\infty} E_{x}^{e x}\left(y, z, m_{x}\right) \cos \left(\frac{m_{x} \pi}{a} x\right) \\
I_{1}(z) & =\sum_{m_{z}=0}^{\infty} I_{1}\left(m_{z}\right) \cos \left(\frac{m_{z} \pi}{h} z\right) \\
I_{2}(x) & =\sum_{m_{x}=0}^{\infty} I_{2}\left(m_{x}\right) \cos \left(\frac{m_{x} \pi}{a} x\right)
\end{aligned}
$$


Then the $z$ and $x$ components of the scattered fields in the arbitrary point with coordinates $(\mathrm{x}, \mathrm{y}, \mathrm{z})$ inside resonator can be written as follows:

$$
\begin{aligned}
& E_{z}^{s c}(x, y, z) \\
& =\int_{0}^{h} G_{z z}^{E}\left(x, y, z, x_{1}, y_{1}, z^{\prime}\right) I_{1}\left(z^{\prime}\right) d z^{\prime} \\
& \quad+\int_{0}^{a} G_{z x}^{E}\left(x, y, z, x^{\prime}, y_{2}, z_{2}\right) I_{2}\left(x^{\prime}\right) d x^{\prime}
\end{aligned}
$$

$$
\begin{aligned}
& G_{x x}^{E}\left(\vec{r}, \vec{r}_{1}\right)=\frac{4 \eta_{0}}{j k V} \sum_{\substack{m_{x}=0 \\
m_{y}=1 \\
m_{z}=1}}^{\infty} \frac{\varepsilon_{m_{1}}\left(k^{2}-\left(k_{x}^{v}\right)^{2}\right) \cos \left(k_{x}^{v} x_{1}\right) \cos \left(k_{x}^{v} x\right) \sin \left(k_{y}^{v} y_{1}\right) \sin \left(k_{y}^{v} y\right) \sin \left(k_{z}^{v} z_{1}\right) \sin \left(k_{z}^{v} z\right)}{k_{v}^{2}-k^{2}} \\
& G_{x z}^{E}\left(\vec{r}, \vec{r}_{1}\right)=-\frac{4 \eta_{0}}{j k V} \sum_{\substack{m_{x}=1 \\
m_{y}=1 \\
m_{z}=1}}^{\infty} \frac{k_{x}^{v} k_{z}^{v} \cos \left(k_{x}^{v} x\right) \sin \left(k_{x}^{v} x_{1}\right) \sin \left(k_{y}^{v} y\right) \sin \left(k_{y}^{v} y_{1}\right) \sin \left(k_{z}^{v} z\right) \cos \left(k_{z}^{v} z_{1}\right)}{k_{v}^{2}-k^{2}}
\end{aligned}
$$

and $G_{z x}^{E}\left(\vec{r}, \vec{r}_{1}\right)=G_{x z}^{E}\left(\vec{r}_{1}, \vec{r}\right)$.

Then one can write zero boundary conditions for tangential components of the total electric field on the boundary of the first and second wire by a standard way (of course, it is again assumed that the thin-wire approximation is valid):

$$
\begin{aligned}
& E_{z}^{s c}\left(x_{1}, y_{1}+r_{0}, z\right)+E_{z}^{e x}\left(x_{1}, y_{1}+r_{0}, z\right) \\
& \quad=0 \text { - first wire } \\
& E_{x}^{s c}\left(x, y_{2}+r_{0}, z_{02}\right)+E_{x}^{e x}\left(x, y_{2}+r_{0}, z_{2}\right) \\
& \quad=0 \text { - second wire }
\end{aligned}
$$

and, taking into account the orthogonality of the system of cosines functions, one can obtain the system of electrical field integral equations for the two-wire system in the modal representation:

$$
\begin{gathered}
I_{1}\left(m_{z}\right) \cdot\left(\left(k_{z}^{v}\right)^{2}-k^{2}\right) \cdot S_{z z}\left(m_{z}\right)-\sum_{m_{x}=0}^{\infty} I_{2}\left(m_{x}\right) \\
\cdot S_{z x}\left(m_{z}, m_{x}\right)=\frac{j k}{\eta_{0}} E_{z}^{e x}\left(x_{1}, y_{1}, m_{z}\right) \\
I_{2}\left(m_{x}\right) \cdot\left(\left(k_{x}^{v}\right)^{2}-k^{2}\right) \cdot S_{x x}\left(m_{x}\right)-\sum_{m_{z}=0}^{\infty} I_{1}\left(m_{z}\right) \\
\cdot S_{x z}\left(m_{x}, m_{z}\right)=\frac{j k}{\eta_{0}} E_{z, m_{1}}^{e x}\left(y_{2}, z_{2}\right)
\end{gathered}
$$

Here the elements $S_{i j}$ are given by

$$
\begin{aligned}
& S_{z z}\left(x_{1}, y_{1}, r_{0}, m_{z}\right)=\frac{4}{a b} \\
& \cdot \sum_{m_{x}, m_{y}=1}^{\infty} \frac{\sin ^{2}\left(k_{x}^{v} x_{1}\right) \sin \left(k_{y}^{v} y_{1}\right) \sin \left(k_{y}^{v}\left(y_{1}+r_{0}\right)\right)}{k_{v}^{2}-k^{2}}
\end{aligned}
$$

$$
\begin{aligned}
& S_{x x}\left(y_{2}, z_{2}, r_{0}, m_{1}\right)=\frac{4}{b h} \\
& \cdot \sum_{m_{y}, m_{z}=1}^{\infty} \frac{\sin \left(k_{y}^{v} y_{2}\right) \sin \left(k_{y}^{v}\left(y_{2}+r_{0}\right)\right) \sin ^{2}\left(k_{z}^{v} z_{2}\right)}{k_{v}^{2}-k^{2}} \\
& S_{z x}\left(x_{1}, y_{1}, y_{2}, z_{2}, m_{x}, m_{z}\right)=-\frac{4}{b h} \\
& \cdot \sum_{m_{y}=1}^{\infty} \frac{k_{x}^{v} k_{z}^{v} \sin \left(k_{x}^{v} x_{1}\right) \sin \left(k_{y}^{v} y_{1}\right) \sin \left(k_{y}^{v} y_{2}\right) \sin \left(k_{z}^{v} z_{2}\right)}{k_{v}^{2}-k^{2}} \\
& S_{x z}\left(x_{1}, y_{1}, y_{2}, z_{2}, m_{x}, m_{z}\right)=-\frac{4}{a b} \\
& \cdot \sum_{m_{y}=1}^{\infty} \frac{k_{x}^{v} k_{z}^{v} \sin \left(k_{x}^{v} x_{1}\right) \sin \left(k_{y}^{v} y_{1}\right) \sin \left(k_{y}^{v} y_{2}\right) \sin \left(k_{z}^{v} z_{2}\right)}{k_{v}^{2}-k^{2}}
\end{aligned}
$$

During the calculation of matrices $S$ summation over one index can be carried out analytically (like in (15) and (16)).

Note that, unlike the case of two parallel wires, considered in the previous section, the amplitudes of current modes for different wire couple with each other; i.e., the system of equations (34a) and (34b) does not split. By this way, to solve this system two methods can be proposed.

The first method, analytical, is an iteration approach. Due to the lack of space in this paper, we will only briefly describe this method. On the first step, the nondiagonal terms in (34a) and (34b) are moved in the right side. Then, as the zero iteration one can consider the noninteracting wires, i.e., assuming that the nondiagonal terms equals zero. Then, each next iteration includes these nondiagonal terms with current modes of previous iteration as sources.

The second method, more numerical, reduces the equation to the linear system for current amplitudes. Restricting the summation by some index $\mathrm{M}_{\max }$ (the calculations have 


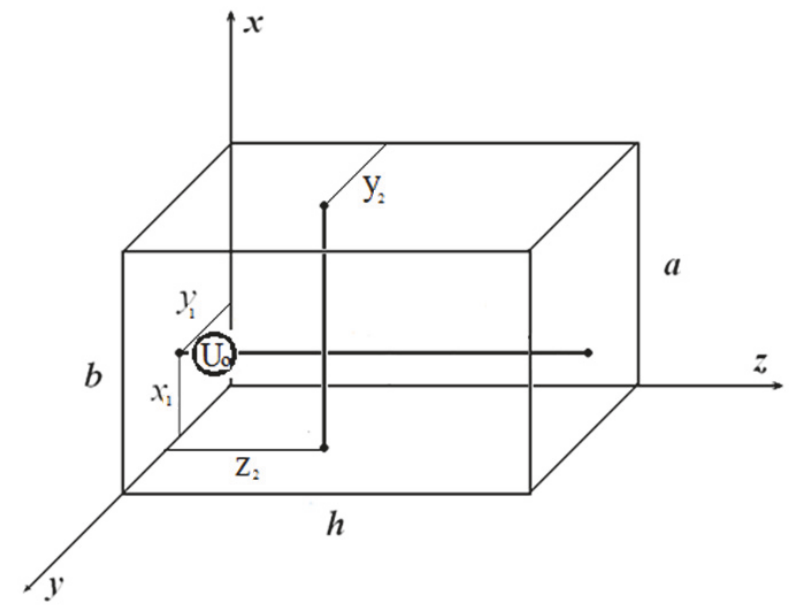

FIGURE 11: Perpendicular wires with symmetrical geometry in resonator.

shown that the value $M_{\max }=500$ describes the current with good accuracy), one can obtain a linear system of the $2 \mathrm{M}_{\max } \times$ $2 \mathrm{M}_{\max }$ order:

$$
\left[\begin{array}{ll}
A_{11} & A_{12} \\
A_{21} & A_{22}
\end{array}\right] \cdot\left[\begin{array}{l}
I_{1} \\
I_{2}
\end{array}\right]=\frac{j k}{\eta_{0}}\left[\begin{array}{c}
E_{1 z}^{\text {ext }} \\
E_{2 z}^{\text {ext }}
\end{array}\right]
$$

where we use the following notations for the $M_{\max } \times M_{\max }$ matrices and $\mathrm{M}_{\max }$-dimension column vectors:

$$
\begin{aligned}
\mathbf{A}_{11} & =\operatorname{diag}\left[\left(\left(k_{z}^{v}\right)^{2}-k^{2}\right) S_{z z}\left(m_{z}\right)\right] \\
\mathbf{A}_{\mathbf{1 2}} & =-\left[S_{z x}\left(m_{z}, m_{x}\right)\right] \\
\mathbf{A}_{22} & =\operatorname{diag}\left[\left(\left(k_{x}^{v}\right)^{2}-k^{2}\right) S_{x x}\left(m_{x}\right)\right] \\
\mathbf{A}_{21} & =-\left[S_{x z}\left(m_{x}, m_{z}\right)\right] \\
\mathbf{I}_{1} & =\left[I_{m_{z}}^{(1)}\right]^{T}, \\
\mathbf{I}_{2} & =\left[I_{m_{x}}^{(2)}\right]^{T}, \\
\mathbf{E}_{\mathbf{1 z}}^{\text {ex }} & =\left[E_{z m_{z}}^{e x}\left(x_{1}, y_{1}\right)\right]^{T}, \\
\mathbf{E}_{2 x}^{\text {ex }} & =\left[E_{x m_{x}}^{e x}\left(y_{2}, z_{2}\right)\right]^{T}
\end{aligned}
$$

By this way, the solution of (36) yields currents induced in the short-circuited perpendicular lines with symmetrical geometry in rectangular resonator by arbitrary (lumped or distributed) sources. The loads at the terminals of the line can be introduced by the usual way, as controlled voltage sources, as it was described in Section 2.2.

The numerical results for the deterministic lines are presented in Figures 12 and 13. The parameters of the resonator are the same, as in the previous examples. The parameters of the lines are as follows: conductor 1 is along $\mathrm{z}$-direction: $\mathrm{x}_{1}=9$ $\mathrm{cm}, \mathrm{y}_{2}=37 \mathrm{~cm}$; conductor 2 is along $\mathrm{x}$-direction: $\mathrm{y}_{2}=0.6 \mathrm{~m}$,

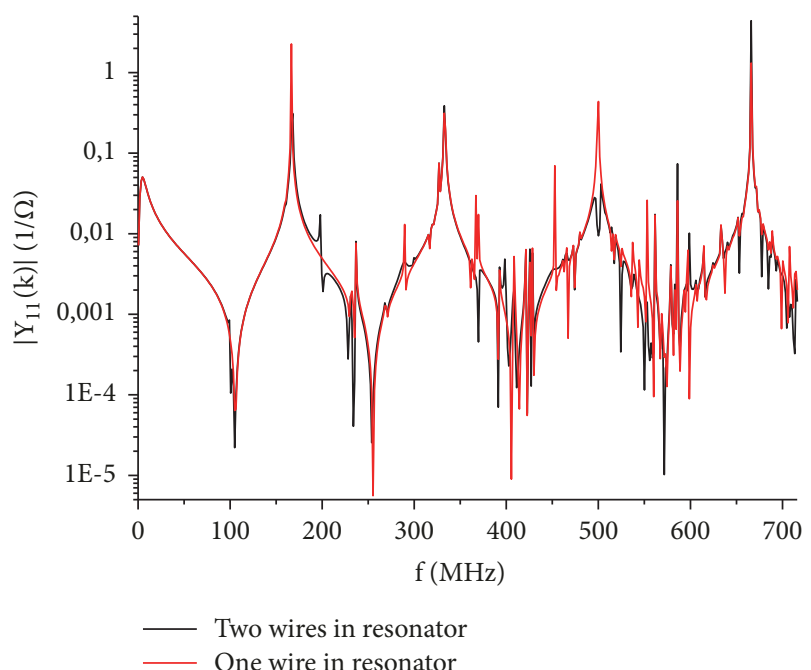

Figure 12: Current in the first, active wire at the point $\mathrm{z}=0$ with and without the second, receiving wire.

$\mathrm{z}_{2}=0.4 \mathrm{~m}$. The first line is excited by a lumped source with unit amplitude at the point $\mathrm{z}=0$. Both lines are short-circuited. The radiuses of the both lines are $1 \mathrm{~mm}$.

The investigation of the perpendicular wires in resonator allows making the following conclusions:

(1) Our method and numerical calculations have a satisfactory agreement for the considered system of two wires.

(2) Far from the cavity resonances the influence of the second wire on the first one is quite small. The current in the first wire can be described by usual TL approximation (see Figure 12). At the same time, near the resonances the influence of the second wire is essential and response of the first wire alone and in the presence of the second one is strongly different.

(3) Far from the cavity resonances $\left(\left|\omega-\omega_{v}\right| \ll \Delta \omega\right.$, where value $\Delta \omega$ is a shift of resonator eigenfrequency caused by the presence of transmission lines in resonator; for the case of one wire in resonator it can be evaluated as $\Delta \omega_{v} \sim$ $\left.\left(4 \pi \sin ^{2}\left(k_{x}^{v} x_{0}\right) \sin ^{2}\left(k_{y}^{v} y_{0}\right) / a b k_{v}^{2} \ln \left(2 x_{0} / r_{0}\right)\right) \omega_{v}\right)$ the current in the second (receive wire) is smaller in comparison with the current in the first one, but near the resonances they can have the same order of magnitude.

(4) The influence of the cavity is essential in the frame of the width of the resonance $\Delta \omega$ (in the system cavity + two wires). For the large number of resonances the distance between them becomes smaller, but the widths of resonances are the same. By this way, the cavity defines the frequency dependence of coupling for large frequencies.

3.2. Randomly Located Perpendicular Wires. Using the developed method one can investigate (within a reasonable time) the statistical properties of induced currents and fields for the case of stochastically arranged symmetrical lines in rectangular resonators. The results of such investigations are presented in Figures 14-17. As in the previous case, the positions of both lines were arranged stochastically: 

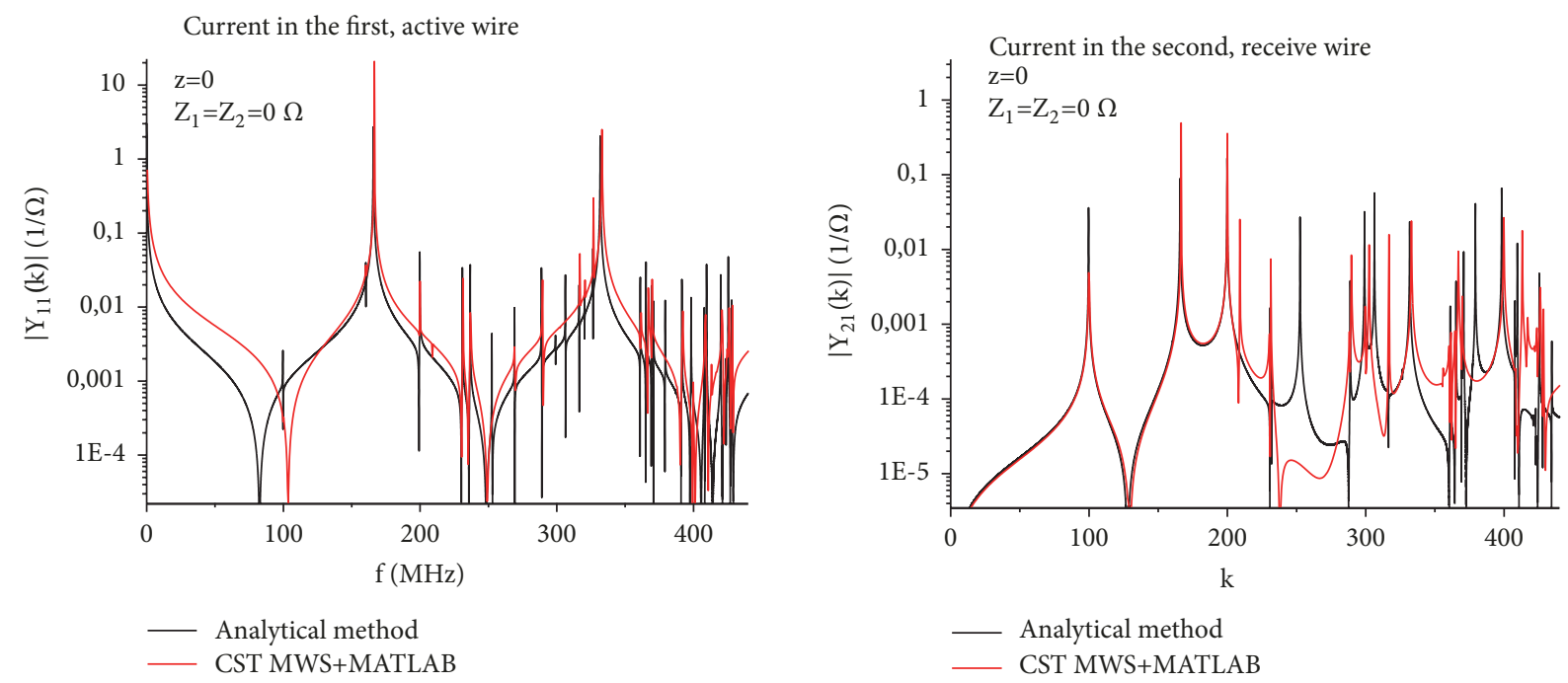

FIGURE 13: Comparison of the currents in the perpendicular wires at the points $\mathrm{z}=0$ and $\mathrm{x}=0$, correspondingly.

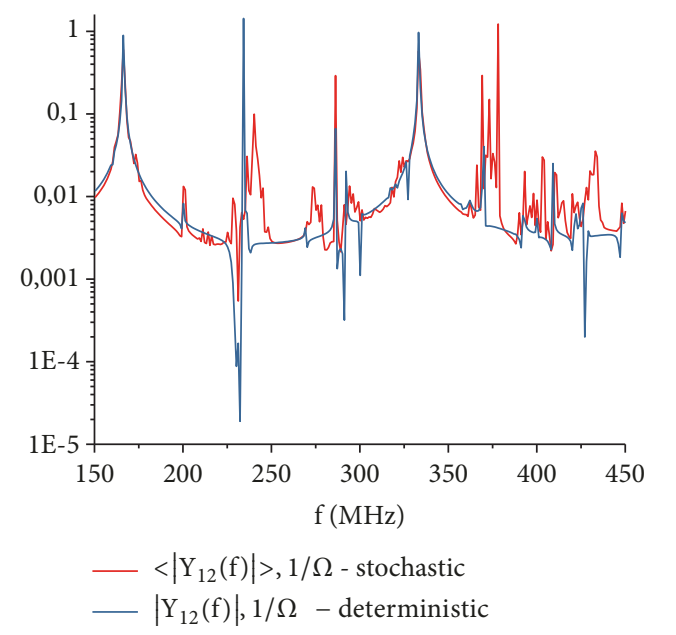

FIGURE 14: Stochastic perpendicular lines in resonator. Averaged response function at the end of the first, active, line compared with the deterministic result.

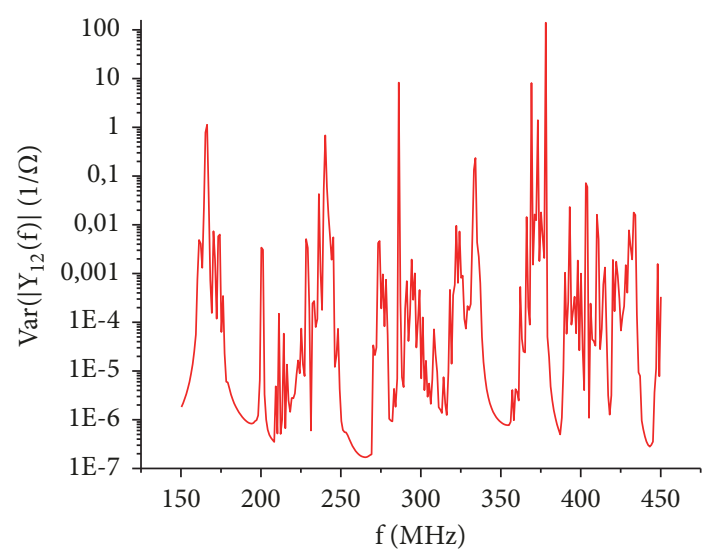

FIGURE 15: Stochastic perpendicular lines in resonator. Variance of the response function of the first, active line.

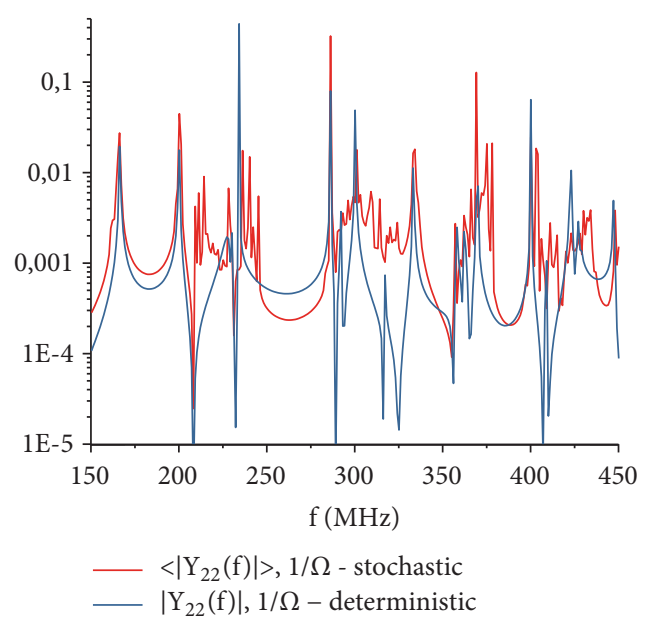

FIGURE 16: Stochastic perpendicular lines in resonator. Averaged response function at the end of the second, receiving, line compared with the deterministic result.

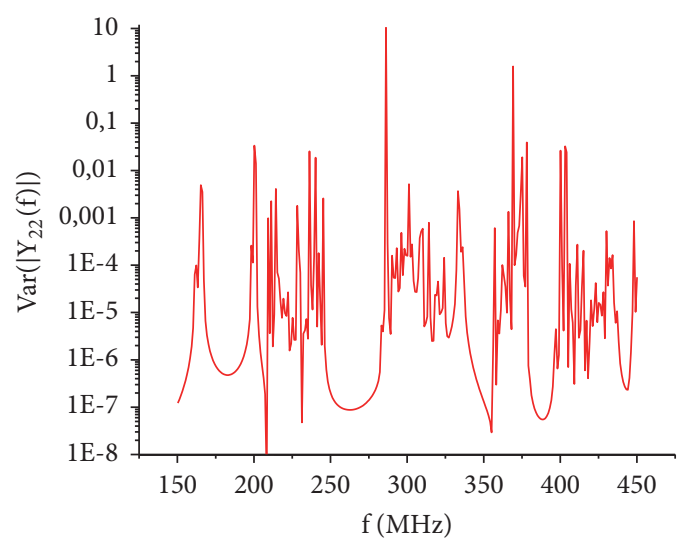

FIGURE 17: Stochastic perpendicular lines in resonator. Variance of the response function of the second, receiving, line. 
$1^{\text {st }}$ transmission line $\| \mathrm{z}$-axis, $\mathrm{x}_{01}=\mathrm{a} \cdot(0.05+0.9 \cdot \mathrm{r})$, $\mathrm{y}_{01}=\mathrm{b} \cdot(0.05+0.9 \cdot \mathrm{r})$

$2^{\text {nd }}$ transmission line $\| x$-axis, $\mathrm{y}_{02}=\mathrm{b} \cdot(0.05+0.9 \cdot \mathrm{r})$, $\mathrm{z}_{02}=\mathrm{h} \cdot(0.05+0.9 \cdot \mathrm{r})$,

where $0<\mathrm{r}<1$ is uniformly distributed stochastic variable. Again, for each frequency point we made 100 statistical events.

Analysis of the curves has shown the next characteristic features. As in the case of parallel wires, the stochastization changes the positions of resonances near the cavity ones, but not the positions of transmission line resonances. For the receiving wire the influence of stochastization is more strong (see Figures 16 and 17). As in deterministic case, the average response of the receiving wire is much smaller (one order of magnitude) than the response of the active wire. At the same time, the statistical variance for the active and receiving wire has the same order of magnitude (Figures 15 and 17).

\section{Conclusion}

In this work the propagation of current waves along stochastic transmission lines inside a resonator was investigated. Two wire configurations were considered: parallel straight wires and perpendicular straight wires. These configurations keep the symmetry of the resonator: the wires connect two opposite walls of the resonator and are parallel to the other four walls. The stochastic line was created by randomizing the positions of the wires. To find the current, an earlier developed method for symmetrical wires inside the resonator was applied for the multiconductor problem. This method gives the general solver for arbitrary excitations, including the considered case of excitation of one wire by a lumped voltage source.

For both geometries it was shown that the influence of the receiving line on the active line is small, when the frequency is far from cavity resonances, but it is essential near these resonances. These effects are kept, for both deterministic and stochastic lines.

The stochastization changes the positions of resonances of the system "wires in resonator" near the cavity ones, but not the positions of transmission line resonances. For the receiving wire the influence of stochastization on the resonant picture is stronger. Unlike the mean value, the statistical variance for the active and receiving wire has the same order of magnitude. Qualitatively the frequency dependency of the variance corresponds to those of paper [8].

The obtained results have several fields of application. They can be used to evaluate the statistical properties of parasitic mutual coupling for the propagation of the signal along a TL in resonators; one can calculate the response of the lines to the external excitation by the EM field penetrating into the resonator through slits and apertures and can evaluate the corresponding statistical properties. Also the results can be used to investigate the damping of the penetrated field by the scattering of the loaded lines (see [19]).

\section{Data Availability}

The data used to support the findings of this study are available from the corresponding author upon request.

\section{Conflicts of Interest}

The authors declare that there are no conflicts of interest regarding the publication of this paper.

\section{References}

[1] S. Hemmady, T. M. Antonsen Jr., E. Ott, and S. M. Anlage, "Statistical prediction and measurement of induced voltages on components within complicated enclosures: A wave-chaotic approach," IEEE Transactions on Electromagnetic Compatibility, vol. 54, no. 4, pp. 758-771, 2012.

[2] X. Zheng, T. M. Antonsen Jr., and E. Ott, "Statistics of impedance and scattering matrices in chaotic microwave cavities: Single channel case," Electromagnetics, vol. 26, no. 1, pp. 3-35, 2006.

[3] J. Yeh, J. A. Hart, E. Bradshaw, T. M. Antonsen, E. Ott, and S. M. Anlage, "Universal and nonuniversal properties of wave-chaotic scattering systems," Physical Review E: Statistical, Nonlinear, and Soft Matter Physics, vol. 81, no. 2, 2010.

[4] A. De Leo, V. M. Primiani, P. Russo, and G. Cerri, "LowFrequency Theoretical Analysis of a Source-Stirred Reverberation Chamber," IEEE Transactions on Electromagnetic Compatibility, vol. 59, no. 2, pp. 315-324, 2017.

[5] I. Junqua, J.-P. Parmantier, and P. Degauque, "Field-to-wire coupling in an electrically large cavity: A semianalytic solution," IEEE Transactions on Electromagnetic Compatibility, vol. 52, no. 4, pp. 1034-1040, 2010.

[6] M. Magdowski, S. V. Tkachenko, and R. Vick, "Coupling of stochastic electromagnetic fields to a transmission line in a reverberation chamber," IEEE Transactions on Electromagnetic Compatibility, vol. 53, no. 2, pp. 308-317, 2011.

[7] M. Magdowski and R. Vick, "Closed-form formulas for the stochastic electromagnetic field coupling to a transmission line with arbitrary loads," IEEE Transactions on Electromagnetic Compatibility, vol. 54, no. 5, pp. 1147-1152, 2012.

[8] L. Nuno, C. L. Holloway, and P. F. Wilson, "Numerical investigation of the induced voltage on a cable placed at random locations inside a metallic enclosure," in Proceedings of the 2008 International Symposium on Electromagnetic Compatibility - EMC Europe, pp. 1-4, Hamburg, Germany, September 2008.

[9] S. Tkachenko, J. Nitsch, and R. Rambousky, "Electromagnetic field coupling to transmission lines inside rectangular resonators," Interaction Notes, vol. 623, 2011, http://www.ece.unm .edu/summa/notes/In/IN623.pdf.

[10] S. V. Tkachenko, R. Rambousky, and J. B. Nitsch, "Electromagnetic field coupling to a thin wire located symmetrically inside a rectangular enclosure," IEEE Transactions on Electromagnetic Compatibility, vol. 55, no. 2, pp. 334-341, 2013.

[11] F. Sabath and E. L. Mokole, "Electromagnetic Coupling to Transmission Lines with Symmetric Geometry Inside Rectangular Resonators," in Ultra-Wideband, Short-Pulse Electromagnetics, F. Sabath and E. L. Mokole, Eds., vol. 10, pp. 31-48, Springer, New York, NY, USA, 2014.

[12] R. Rambousky, S. Tkachenko, and J. Nitsch, "Calculation of currents induced in a long transmission line placed symmetrically inside a rectangular cavity," in Proceedings of the 2013 IEEE International Symposium on Electromagnetic Compatibility EMC 2013, pp. 796-801, Denver, CO, USA, August 2013.

[13] S. Tkachenko, J. Nitsch, R. Vick, and R. Rambousky, "High Frequency Coupling with Stochastic Transmission Line in Rectangular Resonator," in Proceedings of the Symposium 
ASIAEM, Bangalore, India, 2017, http://ece-research.unm.edu/ summa/images/ASIAEM2017/ASIAEM2017abstracts.pdf.

[14] S. V. Tkachenko, H. Scheibe, X. Wang, and R. Vick, "Propagation of current waves along a transmission line with randomly located non-uniformities," in Proceedings of the 2013 International Conference on Electromagnetics in Advanced Applications (ICEAA), pp. 1286-1289, Torino (Turin), Italy, September 2013.

[15] J. B. Nitsch, S. V. Tkachenko, and S. Potthast, "Transient excitation of rectangular resonators through electrically small circular holes," IEEE Transactions on Electromagnetic Compatibility, vol. 54, no. 6, pp. 1252-1259, 2012.

[16] D. A. Hill, "Electronic Mode Stirring for Reverberation Chambers," IEEE Transactions on Electromagnetic Compatibility, vol. 36, no. 4, pp. 294-299, 1994.

[17] A. P. Prudnikov, Y. A. Brychkov, and O. I. Marichev, Integrals and Series, vol. I: Elementary Functions, OPA, Amsterdam, The Netherlands, 1981.

[18] “CST MICROWAVE STUDIO - Providing Complete Technology for High Frequency 3D EM Field Simulation," https://www .cst.com/products/cstmws/solvers.

[19] C. E. Baum, "Damping Electromagnetic Resonances in Equipment Cavities," Interaction Notes, vol. 613, 2010, http://eceresearch.unm.edu/summa/notes/In/0613.pdf. 


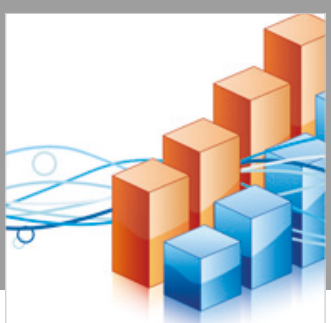

Advances in

Operations Research

\section{-n-m}
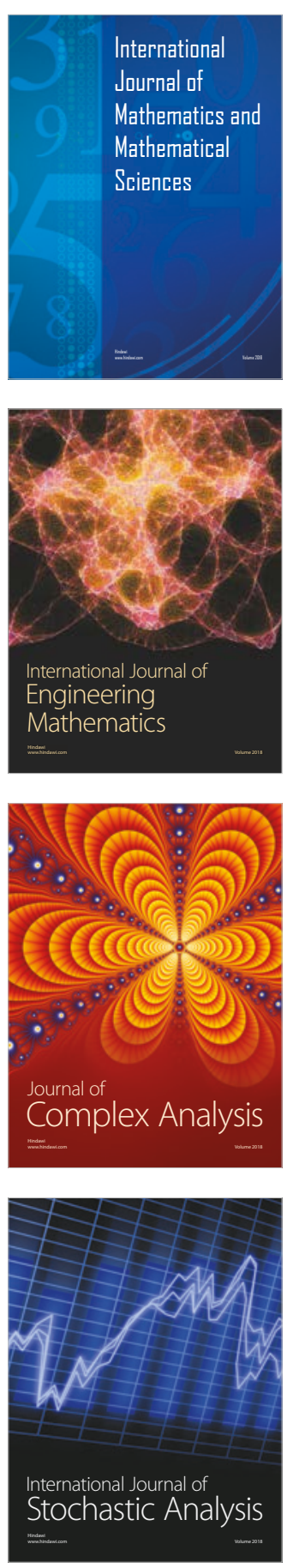
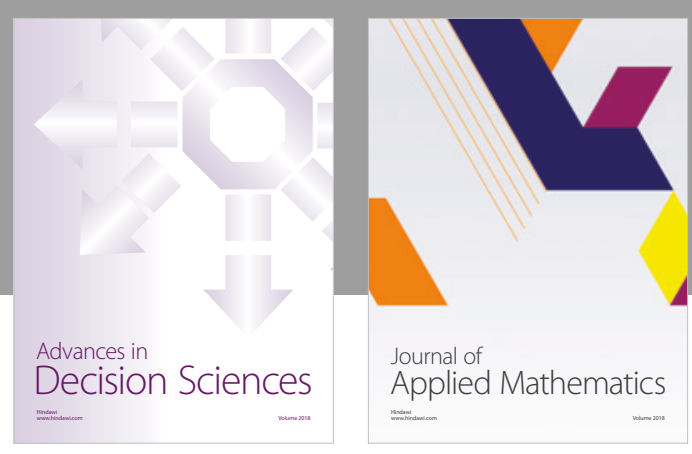

Journal of

Applied Mathematics
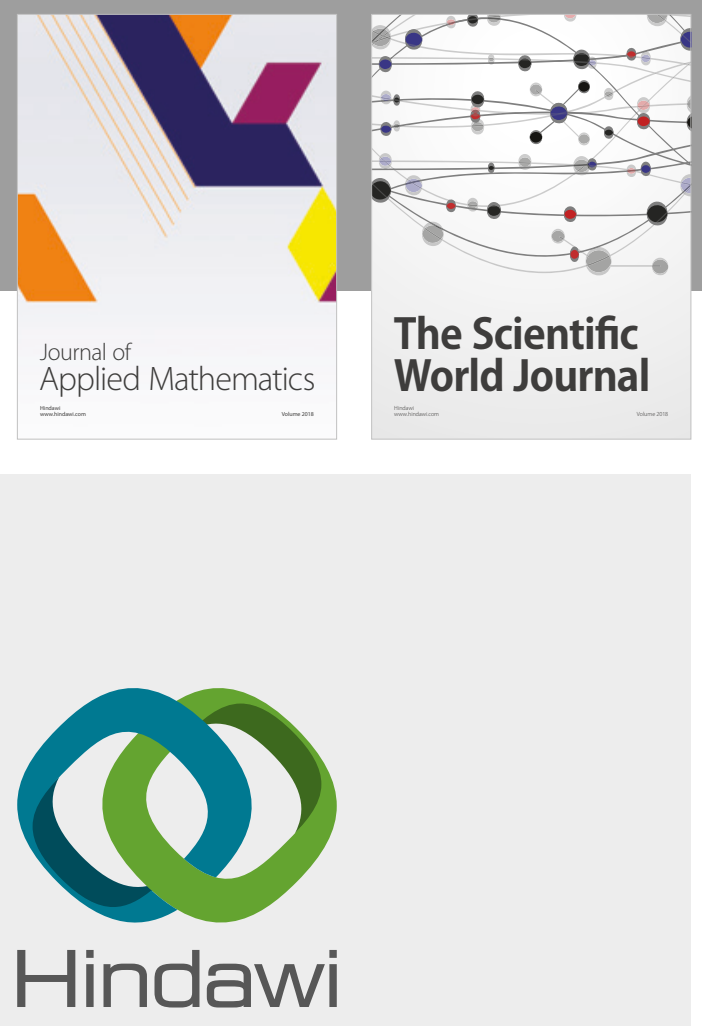

Submit your manuscripts at

www.hindawi.com

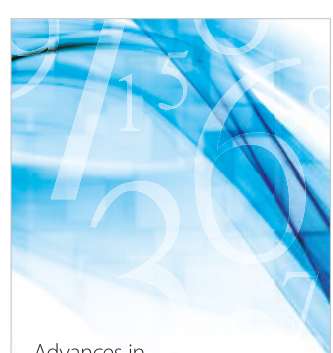

Advances in
Numerical Analysis
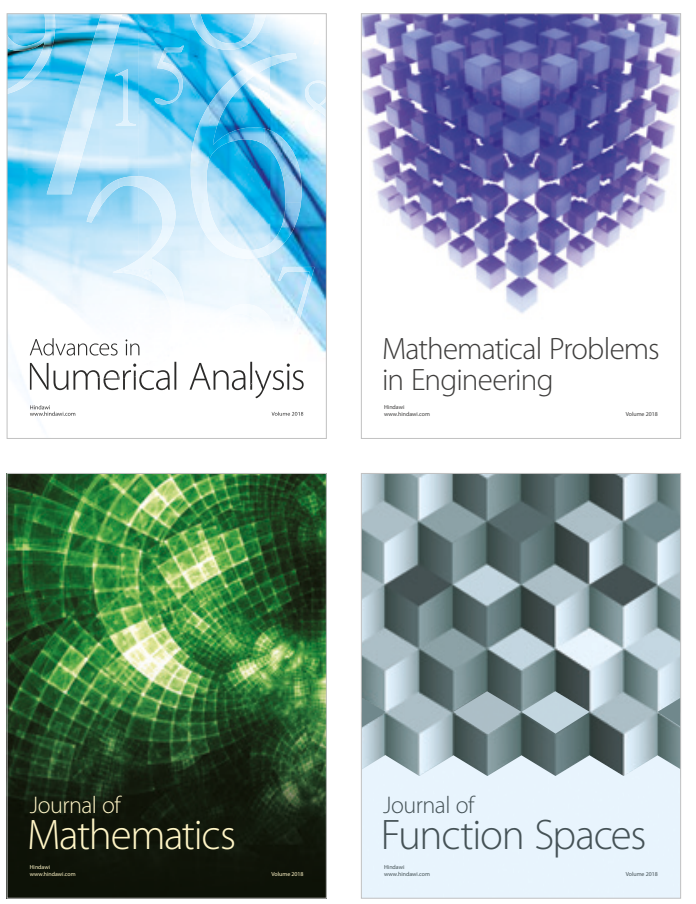

Mathematical Problems in Engineering

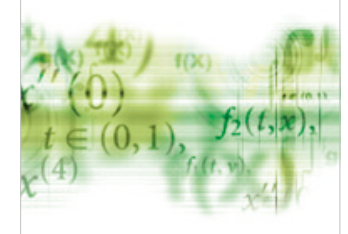

International Journal of

Differential Equations

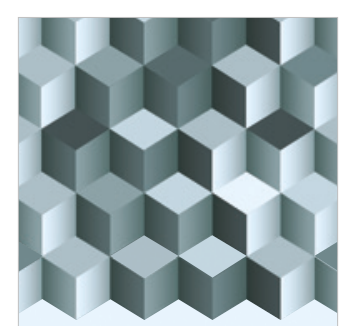

Journal of

Function Spaces

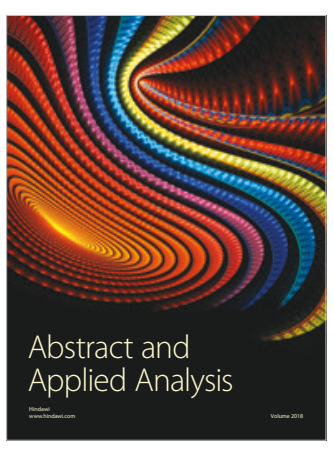

The Scientific

World Journal

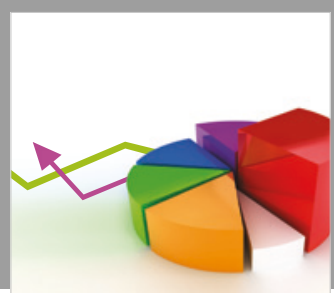

Journal of

Probability and Statistics
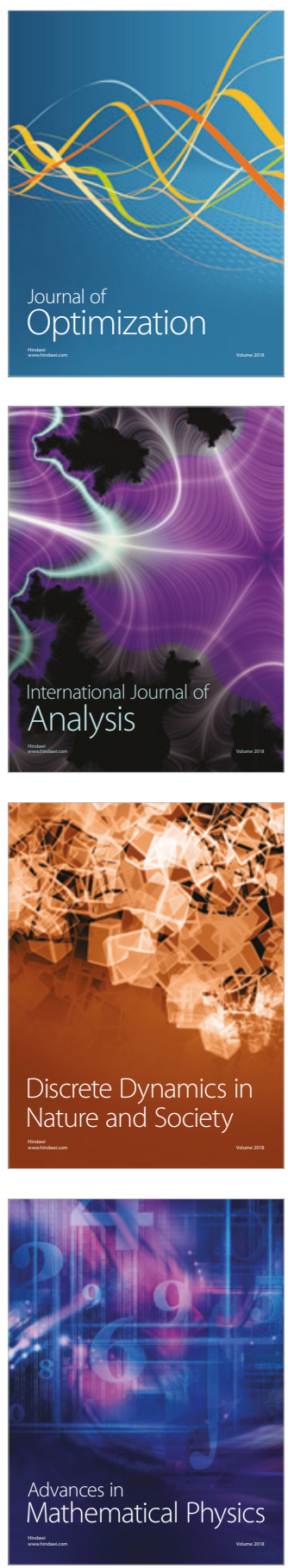